\title{
Imaging the thoracic aorta
}

Evaluation of techniques for aortic imaging is complicated by rapid advances in modern technology and the diverse nature of pathology affecting this region. It is further obfuscated by the assertions of the many single-modality zealots who trade sensitivity and specificity figures like insults. One thing is certain however-no single technique currently offers all the answers to all the questions.

Imaging should quickly, safely, and confidently confirm or refute the presence of aortic disease and determine its extent for treatment planning and prognosis. ${ }^{1}$ A more prosaic but no less serious consideration is to present the thoracic surgeon with images that he or she feels comfortable with. This will obviously differ with local surgical preference, some choosing to operate on the basis of ultrasound findings alone while others will not commit a patient to intervention without a cineangiogram.

The nature of the suspected aortic pathology and the clinical presentation will dictate how the patient is investigated almost as much as the availability of in-house equipment and local expertise. Acute processes such as dissection, rupture of an atheromatous aneurysm, or trauma demand rapid and accurate imaging (preferably at the presenting hospital), whereas the incidental discovery of a coarctation, for example, will allow a more comprehensive response.

\section{Acute aortic disease}

The urgency of presentation and potential instability of the condition of patients with acute aortic dissection effectively narrow the imaging options to angiography, computed tomography (CT), and transoesophageal echocardiography (TOE). The few centres which have developed rapid response magnetic resonance imaging (MRI) protocols can justifiably advocate its primary use in this setting. ${ }^{2}$ Generally speaking, however, MRI is not readily available and the acutely ill patient unquestionably appears more vulnerable inside an eight ton magnet.

Angiography, long considered the gold standard, has been pushed into a niche by TOE and CT, largely because of its invasive nature and inability to detect dissecting haematomas where there is no intimal flap. ${ }^{3}$ To consider angiography defunct, however, reveals a näiveté about the subtlety and complexity of aortic disease. Angiography is useful where there is persisting uncertainty over data from other tests and for those surgeons who prefer the familiar anatomy of biplane arteriograms. There is no question that involvement of branch vessels is more clearly resolved by this technique. Visualisation of the carotid arteries may be desirable if the whole arch is to be replaced, although whether coronary angiography is necessary in the acute setting is debatable.

Transthoracic echocardiography may prove useful in assessing the ascending aorta but it is severely limited distally. In experienced hands, TOE is capable of precise diagnosis of dissection and has the bonus of giving additional information about intracardiac function. ${ }^{4}$ Images produced by this novel technique often show exquisite detail and are capable of convincing the most critical sceptic (fig 1). As a semi-invasive technique TOE is not without risk in the unstable patient but probably has less potential than angiography for causing mishap. Although it compares favourably with other techniques, TOE is not foolproof and reservations have been expressed about blind areas in the ascending aorta and arch produced by the major airways and by considerable vascular unfolding. ${ }^{5}$ These considerations are much less important than the serious issue of availability. Ultrasound is generally accepted as one of the most operator dependent imaging techniques, and it is therefore important for an out of hours service to be manned by experienced operators. Maintenance of this level of expertise will be most difficult to achieve in district general hospitals, and may also cause problems in some tertiary centres. I think that every major
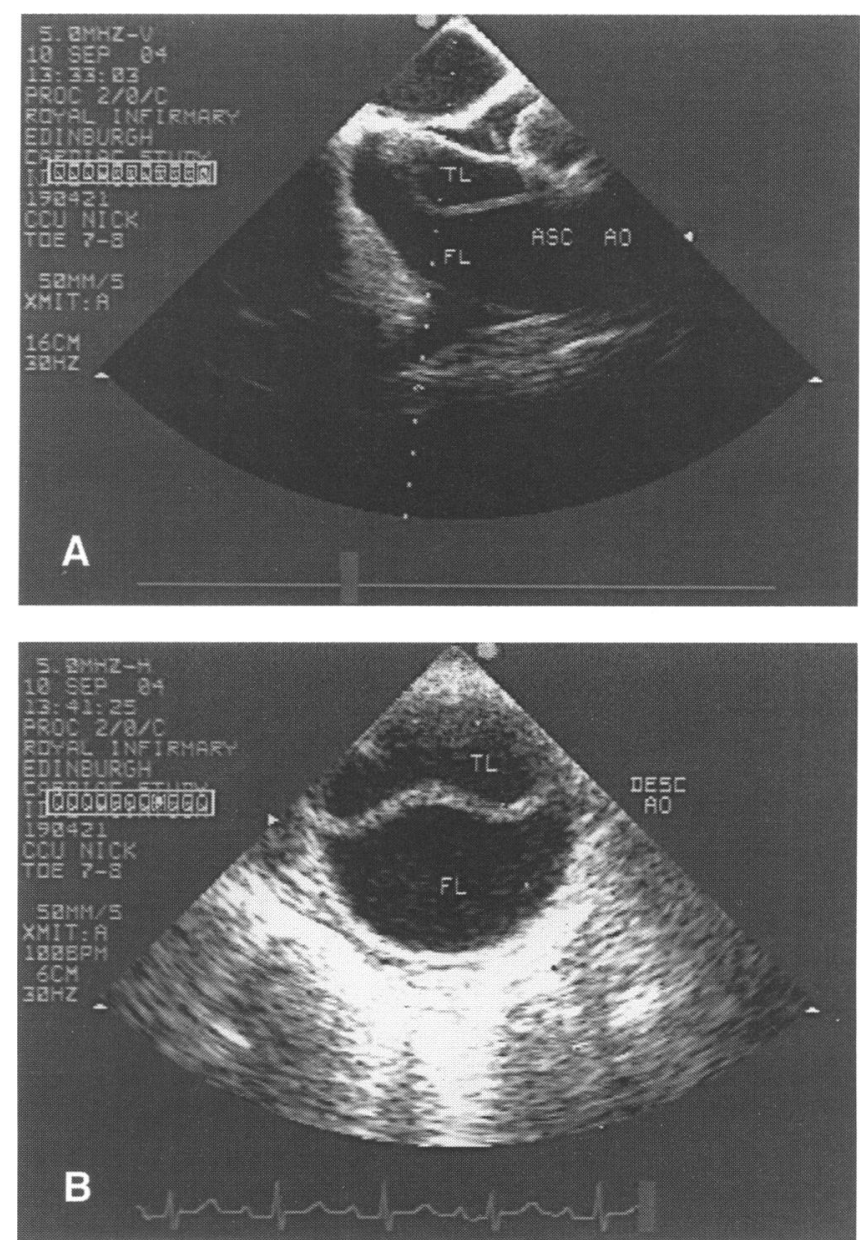

Figure 1 Cross sectional transoesophageal echocardiograms of a type $A$ aortic dissection. The thickened intimal flap and compression of the true lumen $(T L)$ by the false lumen (FL) are well demonstrated in both the ascending aorta $(A)$ and the proximal descending aorta $(B)$. (Courtesy of $\operatorname{Dr} N E R$ Goodfield, Royal Infirmary Edinburgh.) 


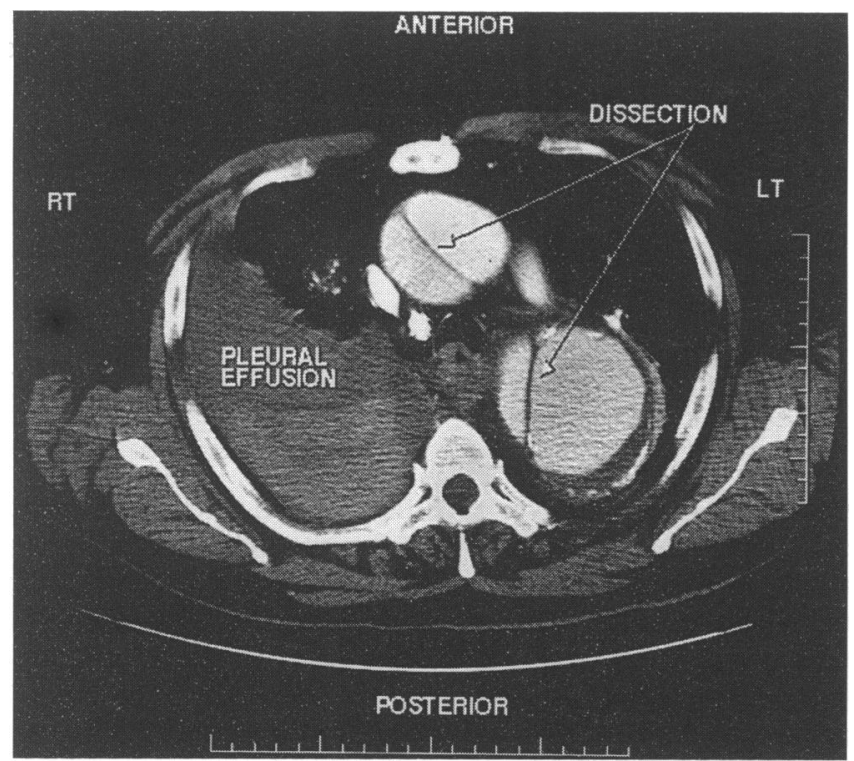

Figurè 2 Contrast enhanced spiral CT image of an acute rupture of a chronic type $A$ dissection. The right sided pleural effusion is in fact a large haemorrhage into the pleura (note the fresh blood causing higher attenuation posteriorly). Aortic wall thickening, intimal flap, and mural calcification are evident. Acquisition time for the whole thoracic aorta was $40 \mathrm{~s}$.

hospital which regularly receives patients with suspected acute aortic disease should have an agreed imaging strategy that operates on a twenty four hour basis. In most instances, this will mean either TOE or CT.

Continuous $x$ ray gantry rotation (spiral CT) has revolutionised CT vascular imaging in the past decade by significantly reducing scan times. Some when they compare other techniques with $\mathrm{CT}$, unashamedly quote results from CT equipment which may be significantly out of date. Many district general hospitals and tertiary centres are beginning to upgrade to spiral acquisition machines that can image the whole contrast enhanced thoracic aorta in less than a minute (fig 2). Reformatting of images used to be a laborious process but is now rapid and user friendly (fig 3). Unfortunately, CT is still unable to offer

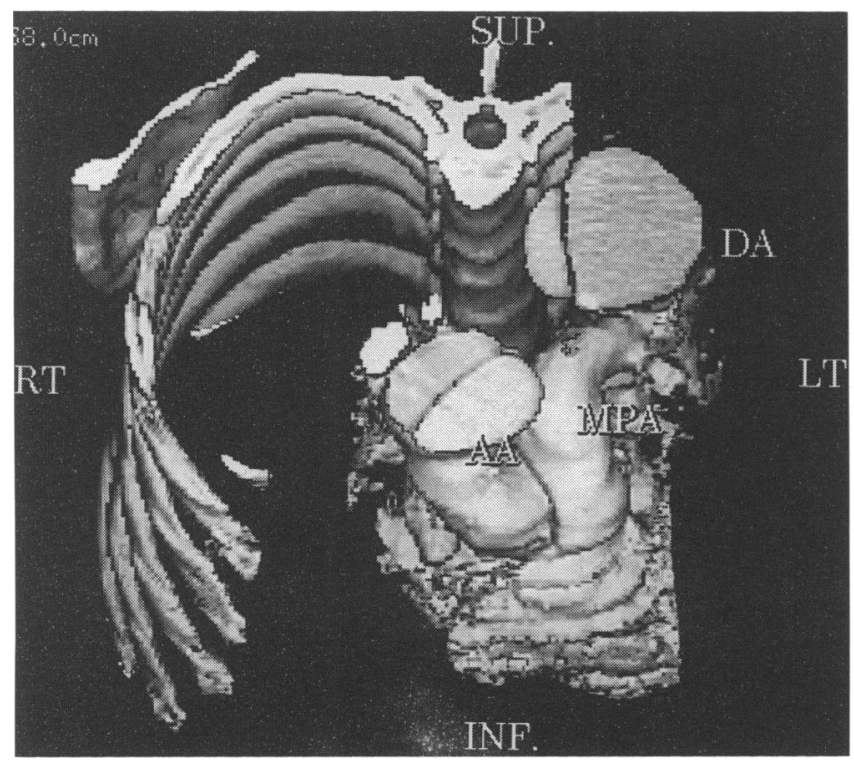

Figure 3 Three dimensional vascular projection of a contrast enhanced spiral CT aortogram (same case as fig 2) showing intimal flap in

ascending and descending aorta. The original model can be viewed in any orientation. The left ribs and sternum have been electronically resected for clarity. MPA, main pulmonary artery; $A A$, ascending aorta; $D A$; descending aorta.

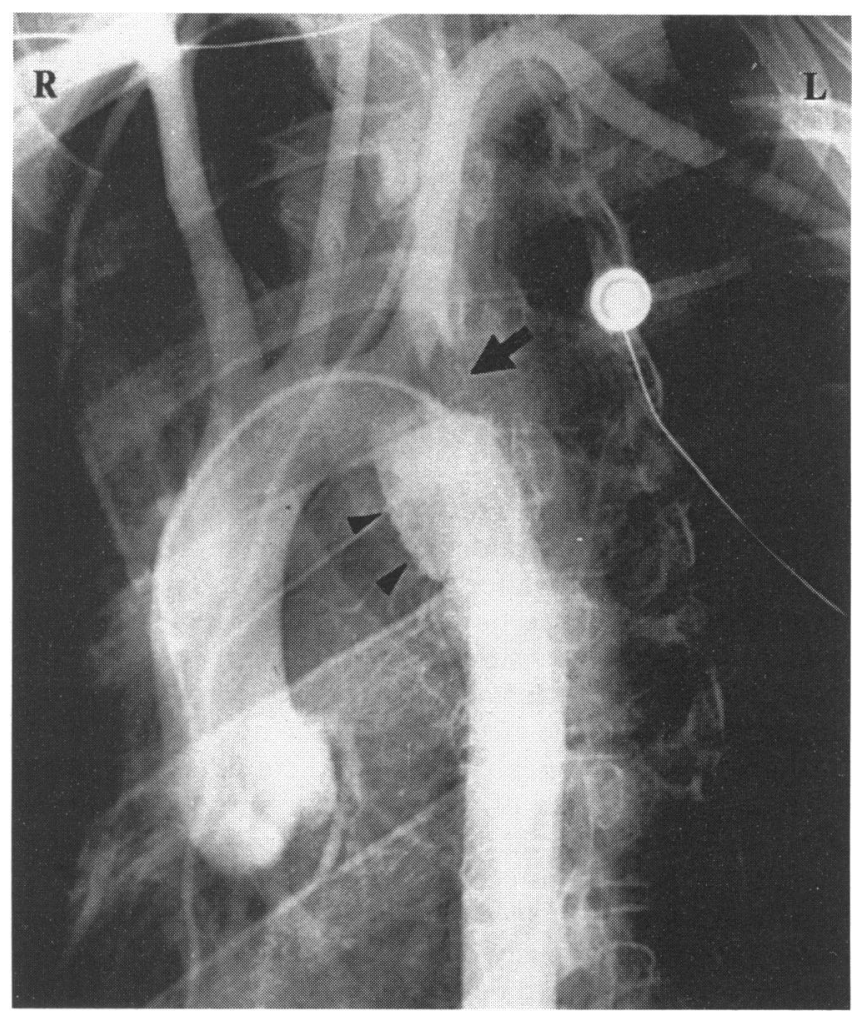

Figure 4 Twenty five year old motorcycle pillion passenger who suffered a major deceleration injury. Left anterior oblique projection of a conventional arch aortogram showing the characteristic localised bulge of a traumatic pseudoaneurysm (arrowheads). The disruption has produced a step in the aorta and that compromises the origin of the left subclavian artery (large arrow).

reliable data on valvar and ventricular function but it compensates for this by providing superior images of para-aortic haematomas, aortic calcification, pleural haemorrhage, and penetrating atheromatous ulcers. ${ }^{6} \mathrm{CT}$ also excels in distinguishing between aortic dissection and other conditions such as invasive lung tumours and oesophageal perforation.

Leaking atheromatous aneurysms of the thoracic aorta are no less serious than dissection and merit equally precise imaging. Associated aortic dilatation, mediastinal haematoma, and pleural effusion are well shown by CT. These aneurysms, however, are frequently accompanied by laminated mural thrombus and consequently CT may not easily distinguish between this entity and a nondissecting intramural haematoma or a thrombosed false lumen. Angiography will be of no help in this situation and so TOE may be the only technique to offer the necessary high resolution tissue characterisation. ${ }^{\text {? }}$

\section{Traumatic aortic disruption}

Most patients who sustain major aortic trauma die before reaching hospital and $40 \%$ of those who initially survive will die in the first 24 hours if untreated. Injury is usually caused by deceleration producing a transverse rupture at the level of the ligamentum arteriosum. False aneurysm formation is frequent and there is a variable amount of periaortic haematoma. Rarely is there any evidence of significant dissection. CT is useful in identifying those patients requiring more definitive investigation, but, being an axial technique it suffers from an intrinsic projectional disadvantage. ${ }^{8}$ Although initial work with TOE is promising, experience is limited in the context of major trauma. ${ }^{9}$ Bulky orthopaedic fixators and life support equipment associated with multiple injuries may render MRI too 


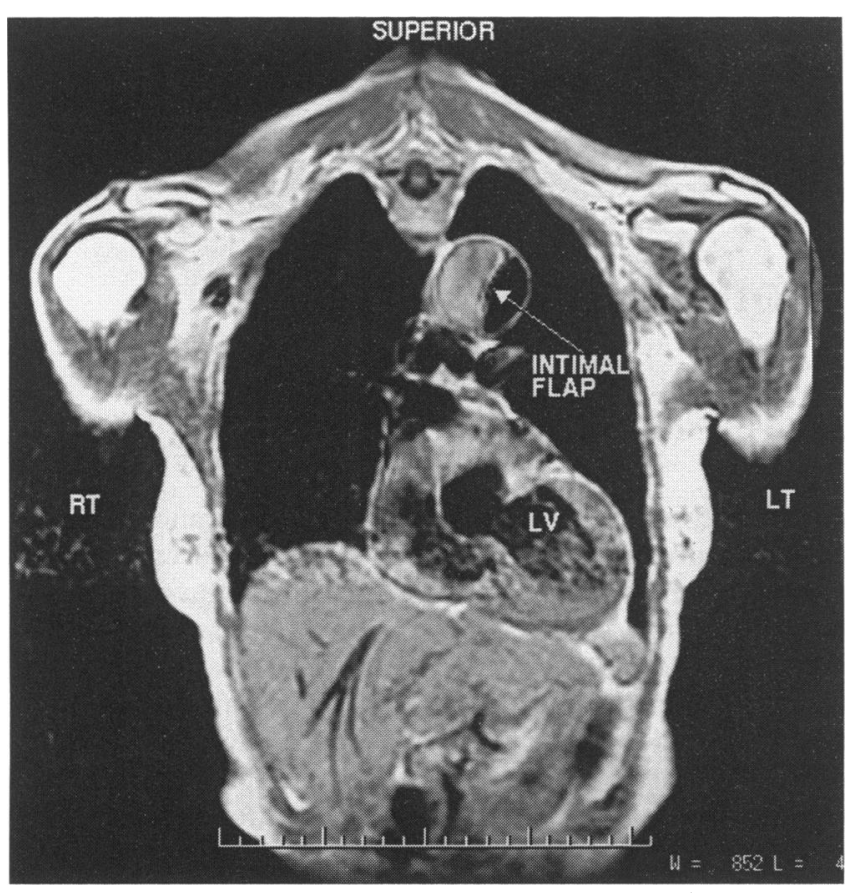

Figure 5 A coronal T1 MRI image of the thorax of a 45 year old woman with a surgically treated type $A$ dissection. Slow turbulent flow in the persistent false lumen shows as the white component of the aortic arch. Faster flow in the true lumen appears black. LV, left ventricle.

cumbersome for general use. Arteriography, therefore, still maintains a prominent role in imaging the trauma patient who has a widened mediastinum ${ }^{10}$ (fig 4 ).

\section{Chronic aortic disease}

Longstanding, stable pathology such as atheromatous or traumatic aneurysm, chronic dissection, annuloaortic ectasia, and coarctation may be imaged satisfactorily by any one of the above techniques. It therefore seems prudent to use the least invasive technique available. This is particularly so when repeated imaging over several years is

Department of Radiology,

The Royal Infirmary of Edinburgh,

Lauriston Place,

Edinburgh EH3 9YW

1 Treasure T. Imaging the dissected aorta. Br Heart $\mathcal{f}$ 1993;70:497-8.

2 Panting JR, Norell MS, Baker C, Nicholson AA. Feasibility, accuracy and safety of magnetic resonance imaging in acute aortic dissection. Clin Radiol 1995;50:455-8.

3 Williams MP, Farrow R. Atypical patterns in the CT diagnosis of aortic dissection. Clin Radiol 1994;49:686-9.

4 Ballal RS, Nanda NC, Gatewood R, D'Arcy B, Samdarshi TE, Holman $\mathrm{WL}$, et al. Usefulness of transesophageal echocardiography in assessment of aortic dissection. Circulation 1991;84:1903-16.

5 Kronzon I, Demopoulos L, Schrem SS, Pasternack P, McCauley D, Freedberg RS, et al. Piffalls in the diagnosis of thoracic aortic aneurysm by Freedberg RS, et al. Pitfalls in the diagnosis of thoracic aortic aneurysm by

6 Kazerooni EA, Bree RL, Williams DM. Penetrating atherosclerotic ulcers of the descending thoracic aorta: evaluation with $\mathrm{CT}$ and distinction

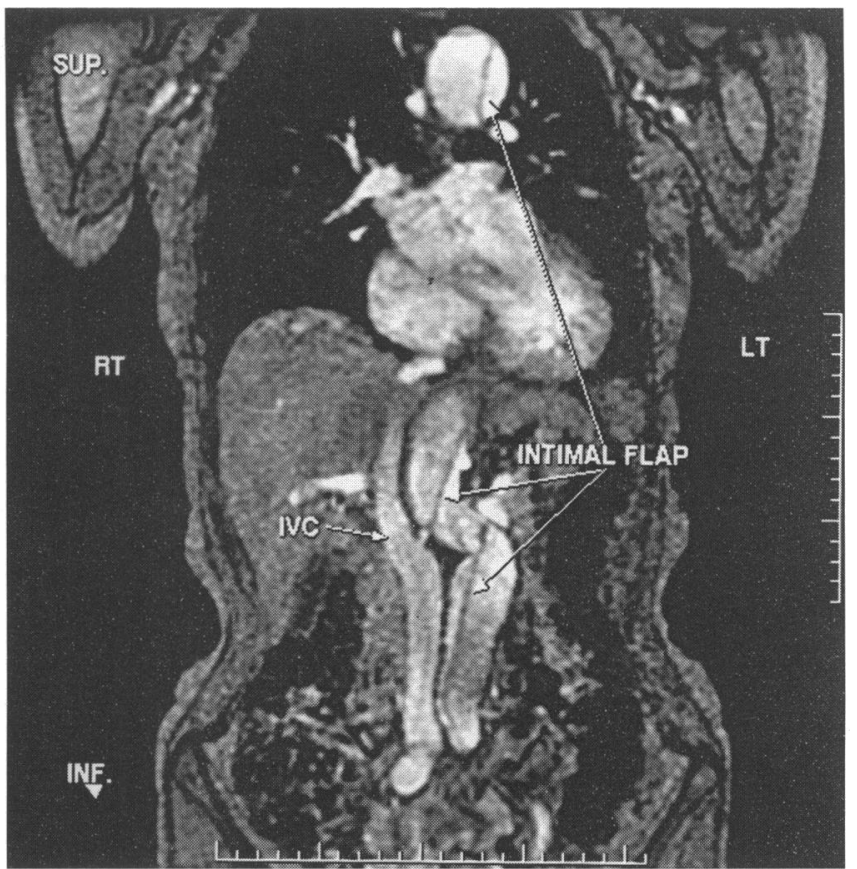

Figure 6 A coronal gradient echo MRI image of the whole torso of patient in fig 5. Note the thin intimal flap extending down the tortuous abdominal aorta to the bifurcation. Flow in both lumens appears white on this type of sequence. This image was acquired in a single 11 second breath hold. IVC, inferior vena cava.

contemplated, as in cases of surgically treated type A dissection where progressive expansion of a persistently patent false lumen requires surveillance. ${ }^{11}$ In this group of chronic conditions, MRI has the unique ability to provide truly non-invasive morphological and flow data in any chosen projection (figs 5 and 6 ).

If recent developments in CT are anything to go by, MRI is set to become considerably faster, less expensive, and more accessible in the near future. I predict that if magnet evolution is maintained there will ultimately be no controversy over which technique is best for aortic imaging.

JOHN H REID

from aortic dissection. Radiology 1992;183:759-65.

7 Taams MA, Gussenhoven WJ, Schippers LA, Roelandt J, van Herwerden LA, Bos E, et al. The value of transesophageal echocardiography for diagnosis of thoracic aorta pathology. Eur Heart $\mathcal{F} 1988 ; 9: 1308-16$.

8 Raptopoulos V, Scheiman RG, Phillips DA, Davidoff A, Silva WE Traumatic aortic tear: screening with chest CT. Radiology 1992;182: 667-73.

9 Moloney JF, Duffy CI, Plehn JF. Transesophageal echocardiographic findings in severe blunt chest trauma (abstract). Circulation 1991;84 suppl ings in severe blunt chest trauma (abstract). Circulation 1991;84 supp

10 White CS, Mirvis SE. Pictorial review: imaging of traumatic aortic injury. Clin Radiol 1995:50:281-7.

11 White R, Ullyot D, Higgins CB. MR imaging of the aorta after surgery for aortic dissection. $A \mathcal{F} R A m \mathcal{F}$ Roentgenol 1988;150:87-92. 